\title{
Impact of Personal Protective Equipment on Intravascular Access Effectiveness
}

\author{
(1) Anna Drozd ${ }^{1}$, (1) Togay Evrin², (1) Dariusz Kosior ${ }^{3}$, (1) Gabriela Borkowska ${ }^{3}$, (1) Sylwia Wozniak ${ }^{4}$, (1) Aleksandra Gasecka ${ }^{5}$, \\ (1) Burak Katipoğlu², (1) Lukasz Szarpak ${ }^{6}$
}

1Polish Society of Disaster Medicine, Warsaw, Poland

2Department of Emergency Medicine, Ufuk University Faculty of Medicine, Dr Rıdvan Ege Training and Research Hospital, Ankara, Turkey

${ }^{3}$ Department of Emergency Medicine, Faculty of Medicine, Collegium Medicum, Cardinal Stefan Wyszynski University, Warsaw, Poland

4 Maria Sklodowska-Curie Medical Academy in Warsaw, Warsaw, Poland

${ }^{5}$ Laboratory of Experimental Clinical Chemistry, Amsterdam University Medical Center, Amsterdam, Netherlands

${ }^{6}$ Maria Sklodowska-Curie Bialystok Oncology Center, Bialystok, Poland

\section{To the Editor,}

A year ago, the world faced the problem of infections with the new SARS-CoV-2 coronavirus, which quickly became a pandemic. The risk of infection for emergency medical personnel is relatively high, so in the era of a pandemic, the best solution would be for medical personnel to provide medical assistance to patients in pre-hospital conditions, when wearing full personal protective equipment (PPE) for aerosol generating procedures (AGPS) $(1,2)$. However, as many studies show, the use of protective suits may reduce the effectiveness of the procedures performed, both in terms of the effectiveness of a procedure and the extension of its time (3-5). In many clinical situations where drugs must be administered quickly or fluid resuscitation is started, prompt intravascular access is a priority for emergency care. However, in the case of a collapsed vascular bed, and the use of prophylactic double gloves, accessing it can be difficult or even impossible. Then an alternative to peripheral intravenous access (PIV) could be intraosseous access (IO). As indicated by many studies, the intraosseous access is a full-fledged intravascular access, through which we can administer both drugs to the patient and use fluid resuscitation or transfuse blood and blood products.
According to the research by Castle et al. (6) the mean length of the procedure for obtaining intraosseous access with PPE AGP was $36 \pm 9.8$ seconds and was statistically significantly shorter than with obtaining intravascular access $(126.9 \pm 39.8 ; p<0.05)$. A similar relationship is also showed by Lamhaut et al. (7), where the procedure time was $65 \pm 17 \mathrm{~s}$ for 10 and $104 \pm 30$ s for PIV, respectively. In turn, Szarpak et al. (8) Comparing the IO access using bone injection gun and Jamshidi with and without PPE AGP, they showed that the use of PPE AGP compared to the procedure without PPE AGP did not significantly extend the duration of the procedure $(29.5 \pm 13.2$ vs $22 \pm 7 \mathrm{~s}, \mathrm{p}=0.063)$. However, accessing with Jamshidi needle was associated with a statistically significantly longer duration of the procedure with PPE-AGP than without PPE-AGP $(69.5 \pm 34.2$ s vs $35 \pm 8 s, p<0.001)$.

In conclusion, as the presented results show, the using of intraosseous punctures as the basic form of intravascular access in the aspect of COVID-19 patients and any other infectious disease is reasonable.

Keywords: Intravascular access, intraosseous access, personal protective equipment, SARS-CoV-2, COVID-19 


\section{Ethics}

Peer-review: Externally peer-reviewed.

\section{Authorship Contributions}

Concept: A.D., T.E., D.K., G.B., S.W., A.G., B.K., L.S., Design: A.D., T.E., D.K., G.B., S.W., A.G., B.K., L.S., Data Collection or Processing: A.D., T.E., D.K., G.B., S.W., A.G., B.K., L.S., Analysis or Interpretation: A.D., T.E., D.K., G.B., S.W., A.G., B.K., L.K., Literature Search: A.D., T.E., D.K., G.B., S.W., A.G., B.K., L.K., Writing: A.D., T.E., D.K., G.B., S.W., A.G., B.K., L.S.

Conflict of Interest: No conflict of interest was declared by the authors.

Financial Disclosure: The authors declared that this study received no financial support.

\section{References}

1. Dzieciatkowski T, Szarpak L, Filipiak KJ, Jaguszewski M, Ladny JR, Smereka J. COVID-19 challenge for modern medicine. Cardiol J. 2020;27:175-83.

2. Korkut S, Evrin T, Katipoglu B, Smereka J, Drozd A, Szarpak L. Resuscitation of Patient with Suspected/Confirmed COVID-19: How to Increase Medical Staff Safety. Eurasian J Emerg Med. 2020;19:184-5.
3. Malysz M, Dabrowski M, Böttiger BW, Smerekaet J, Kulak K, Szarpak A, et al. Resuscitation of the patient with suspected/confirmed COVID-19 when wearing personal protective equipment: A randomized multicenter crossover simulation trial. Cardiol J. 2020;27:497-506.

4. Ludwin K, Bialka S, Czyzewski L, Smereka J, Dabrowski M, Dabrowska A, et al. Video laryngoscopy for endotracheal intubation of adult patients with suspected/ confirmed COVID-19. A systematic review and meta-analysis of randomized controlled trials. Disaster Emerg Med J. 2020;5:85-97.

5. Smereka J, Szarpak L, Filipiak KJ, Jaguszewski M, Ladny JR. Which intravascular access should we use in patients with suspected/confirmed COVID-19? Resuscitation. 2020;151:8-9.

6. Castle N, Owen R, Hann M. Impact of chemical, biological, radiation, and nuclear personal protective equipment on the performance of low- and high-dexterity airway and vascular access skills. Resuscitation. 2009;80:12905.

7. Lamhaut L, Dagron C, Apriotesei R. Comparison of intravenous and intraosseous access by pre-hospital medical emergency personnel with and without CBRN protective equipment. Resuscitation. 2010;81:65-8.

8. Szarpak L, Ramirez JG, Buljan D, Drozd A, Madziała M, Czyzewski L. Comparison of Bone Injection Gun and Jamshidi intraosseous access devices by paramedics with and without chemical-biological-radiological-nuclear personal protective equipment: a randomized, crossover, manikin trial. Am J Emerg Med. 2016;34:1307-8. 